Acta Crystallographica Section E

Structure Reports

Online

ISSN 1600-5368

\section{2-[(2-\{Bis[2-(2-hydroxy-5-nitrobenzyl- ideneamino)ethyl]amino\}ethyl)imino- methyl]-4-nitrophenol acetonitrile monosolvate}

\section{Kwang Ha}

School of Applied Chemical Engineering, The Research Institute of Catalysis, Chonnam National University, Gwangju 500-757, Republic of Korea

Correspondence e-mail: hakwang@chonnam.ac.kr

Received 9 November 2010; accepted 14 November 2010

Key indicators: single-crystal X-ray study; $T=200 \mathrm{~K}$; mean $\sigma(\mathrm{C}-\mathrm{C})=0.005 \AA$; $R$ factor $=0.062 ; w R$ factor $=0.175 ;$ data-to-parameter ratio $=13.6$.

In the title compound, $\mathrm{C}_{27} \mathrm{H}_{27} \mathrm{~N}_{7} \mathrm{O}_{9} \cdot \mathrm{CH}_{3} \mathrm{CN}$, the three nitro groups of the polydentate tripodal Schiff base are located approximately parallel to their respective carrier benzene rings, making dihedral angles of $3.9(4), 5.0(4)$ and $6.3(4)^{\circ}$. Intramolecular $\mathrm{O}-\mathrm{H} \cdots \mathrm{N}$ hydrogen bonds between the hydroxy $\mathrm{O}$ atoms and the imine $\mathrm{N}$ atoms, with $\mathrm{O} \cdots \mathrm{N}$ distances in the range 2.607 (3)-2.665 (3) $\AA$, form nearly planar six-membered rings. In the crystal, weak intermolecular $\mathrm{C}-\mathrm{H} \cdots \mathrm{O}$ and $\mathrm{C}-\mathrm{H} \cdots \mathrm{N}$ hydrogen bonds occur and several intra- and intermolecular $\pi-\pi$ interactions are present between adjacent benzene rings, with a shortest centroidcentroid distance of 3.507 (2) .

\section{Related literature}

For the crystal structure of tris $\{2-[(5$-bromosalicylidene)amino]ethyl $\}$ amine, see: Kanesato et al. (2001).

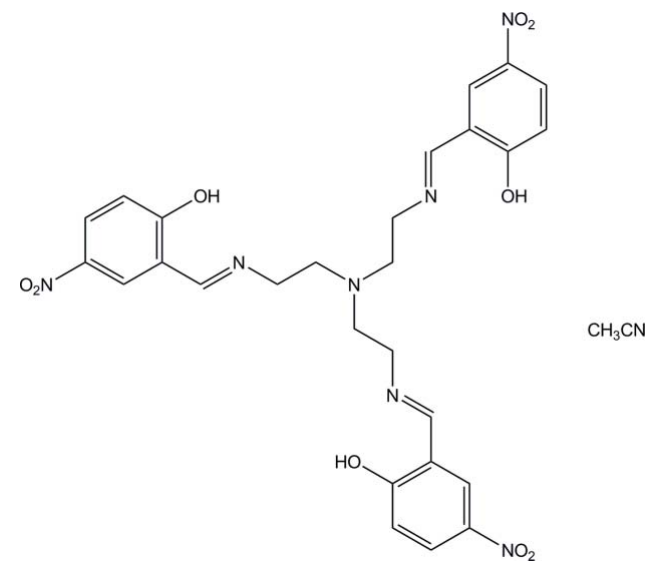

\section{Experimental}

Crystal data

\begin{tabular}{|c|c|}
\hline $\begin{array}{l}\mathrm{C}_{27} \mathrm{H}_{27} \mathrm{~N}_{7} \mathrm{O}_{9} \cdot \mathrm{C}_{2} \mathrm{H}_{3} \mathrm{~N} \\
M_{r}=634.61 \\
\text { Triclinic, } P \overline{1} \\
a=10.6097(9) \AA \\
b=11.8168(9) \AA \\
c=12.8003(10) \AA \\
\alpha=79.054(2)^{\circ}\end{array}$ & $\begin{array}{l}\gamma=88.527(2)^{\circ} \\
V=1462.1(2) \AA^{3} \\
Z=2 \\
\text { Mo } K \alpha \text { radiation } \\
\mu=0.11 \mathrm{~mm}^{-1} \\
T=200 \mathrm{~K} \\
0.32 \times 0.13 \times 0.11 \mathrm{~mm}\end{array}$ \\
\hline
\end{tabular}

$\beta=68.293(2)^{\circ}$

Data collection

Bruker SMART 1000 CCD diffractometer

Absorption correction: multi-scan (SADABS; Bruker, 2000)

$T_{\min }=0.846, T_{\max }=0.988$

9227 measured reflections 5688 independent reflections 3102 reflections with $I>2 \sigma(I)$ $R_{\text {int }}=0.038$

Refinement

$R\left[F^{2}>2 \sigma\left(F^{2}\right)\right]=0.062$

$w R\left(F^{2}\right)=0.175$

$S=1.03$

5688 reflections

419 parameters

$\mathrm{H}$-atom parameters constrained

$\Delta \rho_{\max }=0.41$ e $\AA^{-3}$

$\Delta \rho_{\min }=-0.42$ e $\AA^{-3}$

Table 1

Hydrogen-bond geometry $\left(\AA,^{\circ}\right)$.

\begin{tabular}{lllll}
\hline$D-\mathrm{H} \cdots A$ & $D-\mathrm{H}$ & $\mathrm{H} \cdots A$ & $D \cdots A$ & $D-\mathrm{H} \cdots A$ \\
\hline $\mathrm{O} 1-\mathrm{H} 1 O \cdots \mathrm{N} 2$ & 0.84 & 1.87 & $2.627(3)$ & 149 \\
$\mathrm{O} 4-\mathrm{H} 4 O \cdots \mathrm{N} 4$ & 0.84 & 1.92 & $2.665(3)$ & 147 \\
$\mathrm{O} 7-\mathrm{H} 7 O \cdots \mathrm{N} 6$ & 0.84 & 1.85 & $2.607(3)$ & 149 \\
$\mathrm{C} 1-\mathrm{H} 1 A \cdots \mathrm{N} 8^{\mathrm{i}}$ & 0.99 & 2.56 & $3.369(5)$ & 139 \\
$\mathrm{C} 1-\mathrm{H} 1 B \cdots \mathrm{O} 4^{\mathrm{ii}}$ & 0.99 & 2.41 & $3.290(4)$ & 148 \\
$\mathrm{C} 2-\mathrm{H} 2 B \cdots \mathrm{O} 2^{\text {iii }}$ & 0.99 & 2.44 & $3.300(4)$ & 146 \\
$\mathrm{C} 3-\mathrm{H} 3 \cdots \mathrm{O} 7$ & 0.95 & 2.53 & $3.297(4)$ & 138 \\
$\mathrm{C} 6-\mathrm{H} 6 \cdots \mathrm{N} 8^{\text {iv }}$ & 0.95 & 2.49 & $3.360(5)$ & 153 \\
$\mathrm{C} 9-\mathrm{H} 9 \cdots \mathrm{O} 7$ & 0.95 & 2.55 & $3.328(4)$ & 139 \\
$\mathrm{C} 11-\mathrm{H} 11 A \cdots \mathrm{O} 5^{\mathrm{v}}$ & 0.99 & 2.40 & $3.331(4)$ & 157 \\
$\mathrm{C} 12-\mathrm{H} 12 \cdots \mathrm{O}^{\mathrm{v}}$ & 0.95 & 2.54 & $3.339(4)$ & 142 \\
$\mathrm{C} 16-\mathrm{H} 16 \cdots 6^{\text {vi }}$ & 0.95 & 2.51 & $3.330(4)$ & 145 \\
$\mathrm{C} 25-\mathrm{H} 25 \cdots \mathrm{O}^{\text {vii }}$ & 0.95 & 2.48 & $3.359(4)$ & 153 \\
\hline
\end{tabular}

Symmetry codes: (i) $x+1, y, z$; (ii) $-x+2,-y,-z$; (iii) $-x+2,-y,-z+1$; (iv) $x+1, y-1, z ; \quad$ (v) $\quad-x+1,-y,-z+1 ; \quad$ (vi) $\quad-x+1,-y-1,-z+1$; $\quad$ (vii) $-x+1,-y,-z+2$.

Data collection: SMART (Bruker, 2000); cell refinement: SAINT (Bruker, 2000); data reduction: $S A I N T$; $\operatorname{program}(\mathrm{s})$ used to solve structure: SHELXS97 (Sheldrick, 2008); program(s) used to refine structure: SHELXL97 (Sheldrick, 2008); molecular graphics: ORTEP-3 (Farrugia, 1997) and PLATON (Spek, 2009); software used to prepare material for publication: SHELXL97.

This work was supported by the Priority Research Centers Program through the National Research Foundation of Korea (NRF) funded by the Ministry of Education, Science and Technology (2009-0094056).

Supplementary data and figures for this paper are available from the IUCr electronic archives (Reference: IS2631). 


\section{organic compounds}

\section{References}

Bruker (2000). SADABS, SMART and SAINT. Bruker AXS Inc., Madison, Wisconsin, USA.
Farrugia, L. J. (1997). J. Appl. Cryst. 30, 565.

Kanesato, M., Ngassapa, F. N. \& Yokoyama, T. (2001). Anal. Sci. 17, 471-472.

Sheldrick, G. M. (2008). Acta Cryst. A64, 112-122.

Spek, A. L. (2009). Acta Cryst. D65, 148-155. 


\section{supporting information}

Acta Cryst. (2010). E66, o3222-03223 [https://doi.org/10.1107/S1600536810047185]

\section{2-[(2-\{Bis[2-(2-hydroxy-5-nitrobenzylideneamino)ethyl] amino\}ethyl)imino- methyl]-4-nitrophenol acetonitrile monosolvate}

\section{Kwang Ha}

\section{S1. Comment}

The title compound, $\mathrm{C}_{27} \mathrm{H}_{27} \mathrm{~N}_{7} \mathrm{O}_{9} . \mathrm{CH}_{3} \mathrm{CN}$, consists of a polydentate tripodal Schiff base and an acetonitrile solvent molecule (Fig. 1). The Schiff base can act as a tribasic hexa- or heptadentate ligand, that is, the $\mathrm{N}_{3} \mathrm{O}_{3}$ or $\mathrm{N}_{4} \mathrm{O}_{3}$ donor atoms can coordinate to a metal ion or metal ions. In the crystal structure, the Schiff base reveals an approximate threefold axis, when viewed down the apical amine $\mathrm{N}$ atom (N1) through the plane formed by the atoms $\mathrm{C} 1, \mathrm{C} 10$ and $\mathrm{C} 19$, and three nitro groups are located approximately parallel to their respective carrier benzene rings. The $\mathrm{N}-\mathrm{C}$ bond lengths and the $\mathrm{C}-\mathrm{N}-\mathrm{C}$ bond angles indicate that the apical $\mathrm{N} 1$ atom is $s p^{3}$-hybridized $[d(\mathrm{~N} 1-\mathrm{C})=1.470(4)-1.480(4) \AA ;<\mathrm{C}-\mathrm{N} 1-$ $\left.\mathrm{C}=109.5(2)-111.7(2)^{\circ}\right]$ and the other imine $\mathrm{N}$ atoms $(\mathrm{N} 2, \mathrm{~N} 4, \mathrm{~N} 6)$ are $s p^{2}$-hybridized $[d(\mathrm{~N}=\mathrm{C})=1.291(4)-1.307(4) \AA$ and $\left.d(\mathrm{~N}-\mathrm{C})=1.460(4)-1.469(4) \AA ;<\mathrm{C}-\mathrm{N}-\mathrm{C}=122.7(3)-123.9(3)^{\circ}\right]$. The compound displays strong intramolecular $\mathrm{O}-\mathrm{H} \cdots \mathrm{N}$ hydrogen bonds between the hydroxy $\mathrm{O}$ atoms and the imine $\mathrm{N}$ atoms with $d(\mathrm{O} \cdots \mathrm{N})=2.607$ (3)-2.665 (3) $\AA$ thus forming a nearly planar six-membered ring (Fig. 2, Table 1). There are also weak intermolecular $\mathrm{C}-\mathrm{H} \cdots \mathrm{O}$ and $\mathrm{C}-$ $\mathrm{H} \cdots \mathrm{N}$ hydrogen bonds with $d(\mathrm{C} \cdots \mathrm{O})=3.290(4)-3.359(4) \AA$ and $d(\mathrm{C} \cdots \mathrm{N})=3.360(5)-3.369(5) \AA$. Moreover, several intra- and intermolecular $\pi-\pi$ interactions between the adjacent benzene rings are present, with a shortest ring centroidcentroid distance of 3.507 (2) $\AA$, and the dihedral angle between the ring planes is $5.1(2)^{\circ}$.

\section{S2. Experimental}

Tris(2-aminoethyl)amine (0.7305 g, $4.995 \mathrm{mmol})$ and 5-nitrosalicylaldehyde (2.5077 g, $15.005 \mathrm{mmol})$ in EtOH (30 ml) were stirred for $3 \mathrm{~h}$ at room temperature. The precipitate was then separated by filtration, washed with ether, and dried at

$50{ }^{\circ} \mathrm{C}$, to give a yellow powder $(2.9135 \mathrm{~g})$. Crystals suitable for X-ray analysis were obtained by slow evaporation from a $\mathrm{CH}_{3} \mathrm{CN}$ solution.

\section{S3. Refinement}

$\mathrm{H}$ atoms were positioned geometrically and allowed to ride on their respective parent atoms $[\mathrm{C}-\mathrm{H}=0.95 \AA(\mathrm{CH}), 0.99$ $\AA\left(\mathrm{CH}_{2}\right)$ or $0.98 \AA\left(\mathrm{CH}_{3}\right)$ and $\mathrm{O}-\mathrm{H}=0.84 \AA$, and $U_{\text {iso }}(\mathrm{H})=1.2 U_{\text {eq }}(\mathrm{C})$ or $1.5 U_{\text {eq }}($ methyl $\left.\mathrm{C}, \mathrm{O})\right]$. 


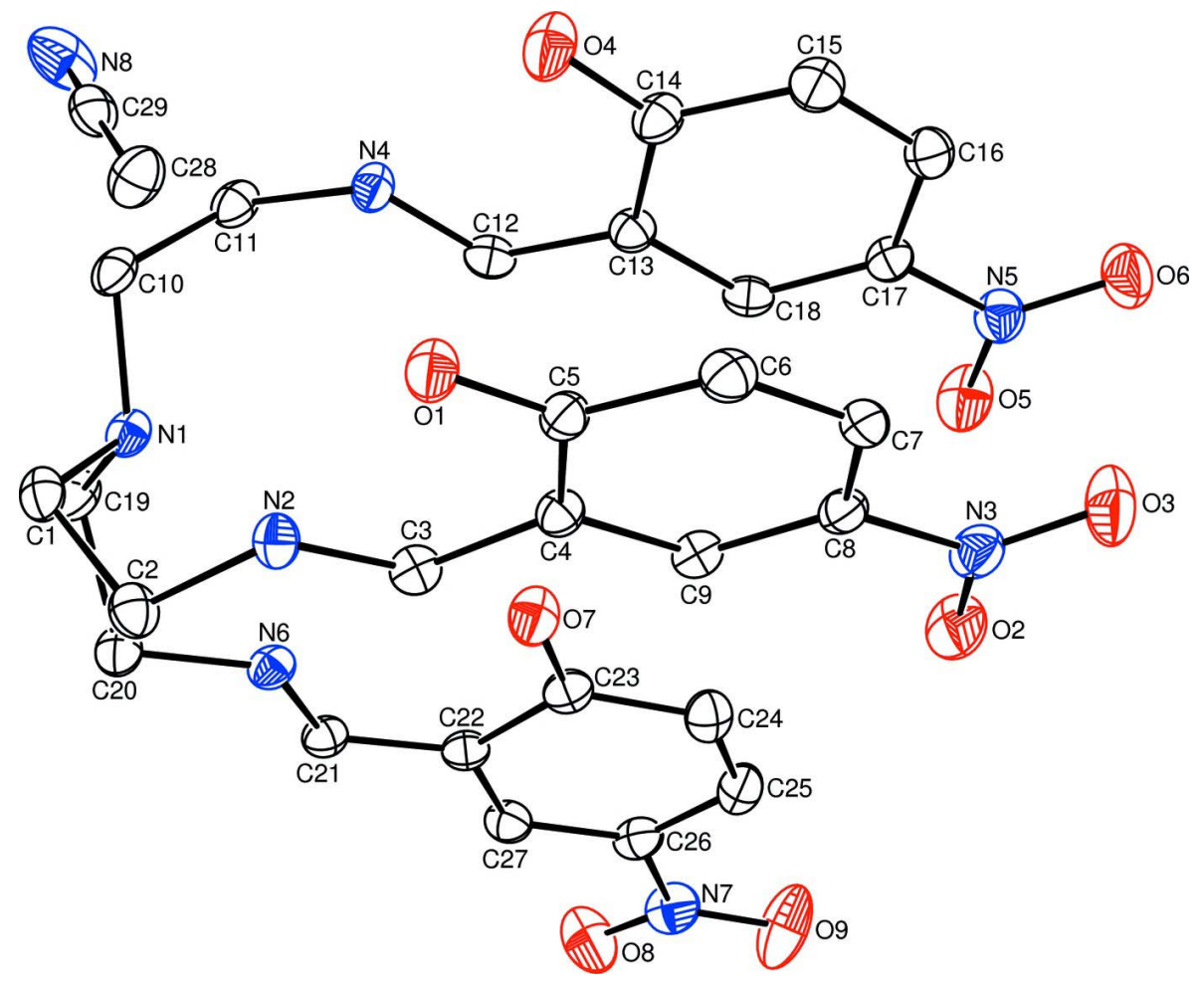

Figure 1

The structure of the title compound, with displacement ellipsoids drawn at the $40 \%$ probability level. $\mathrm{H}$ atoms are omitted for clarity. 


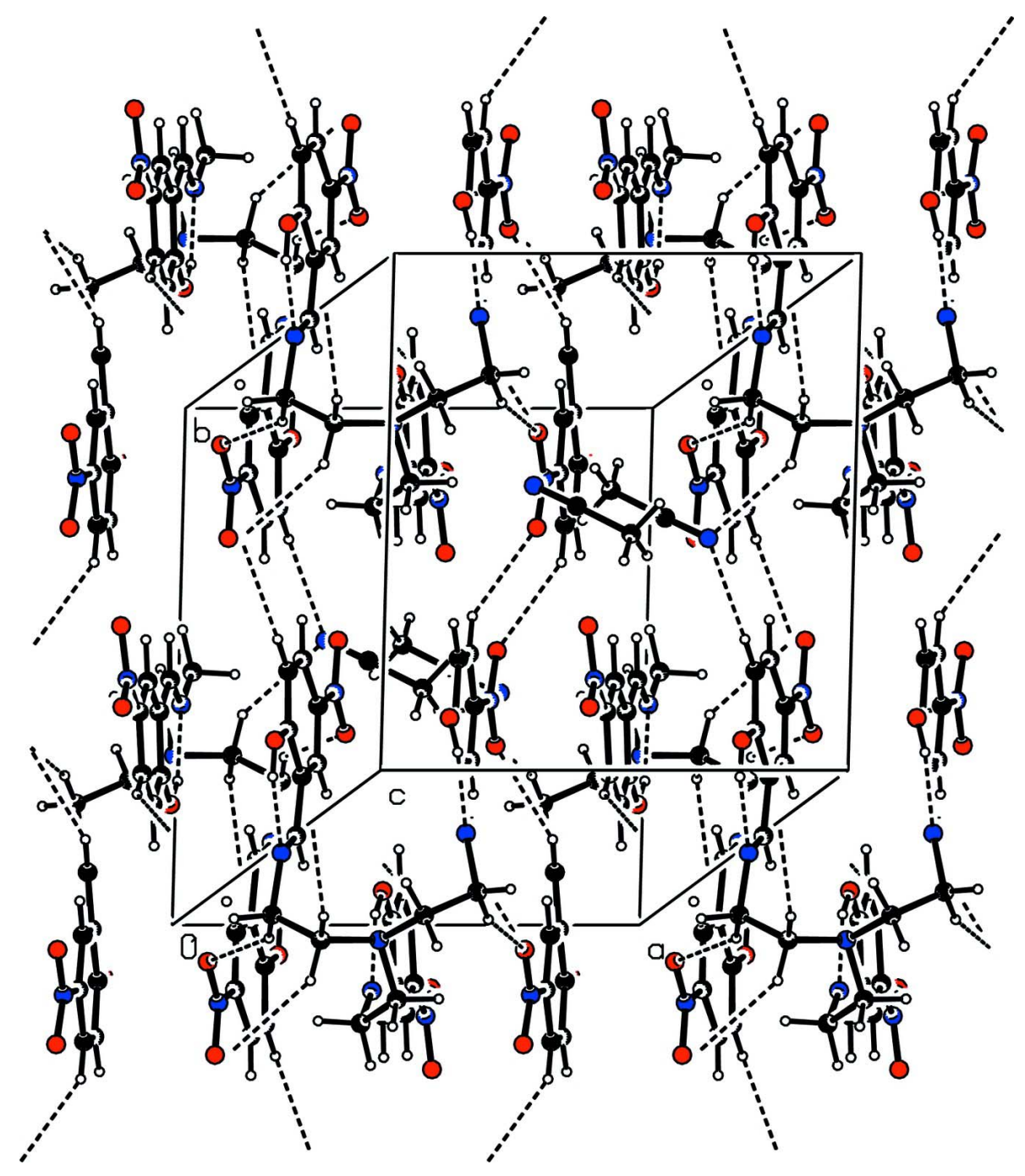

Figure 2

View of the unit-cell contents of the title compound. Hydrogen-bond interactions are drawn with dashed lines.

2-[(2-\{Bis[2-(2-hydroxy-5- nitrobenzylideneamino)ethyl]amino\}ethyl)iminomethyl]-4-nitrophenol acetonitrile monosolvate

Crystal data

$\mathrm{C}_{27} \mathrm{H}_{27} \mathrm{~N}_{7} \mathrm{O}_{9} \cdot \mathrm{C}_{2} \mathrm{H}_{3} \mathrm{~N}$

$M_{r}=634.61$

Triclinic, $P \overline{1}$

Hall symbol: -P 1

$a=10.6097(9) \AA$

$b=11.8168(9) \AA$

$c=12.8003(10) \AA$

$\alpha=79.054(2)^{\circ}$

$\beta=68.293(2)^{\circ}$

$\gamma=88.527(2)^{\circ}$

$V=1462.1(2) \AA^{3}$

\section{$Z=2$}

$F(000)=664$

$D_{\mathrm{x}}=1.441 \mathrm{Mg} \mathrm{m}^{-3}$

Mo $K \alpha$ radiation, $\lambda=0.71073 \AA$

Cell parameters from 2046 reflections

$\theta=2.2-25.2^{\circ}$

$\mu=0.11 \mathrm{~mm}^{-1}$

$T=200 \mathrm{~K}$

Block, yellow

$0.32 \times 0.13 \times 0.11 \mathrm{~mm}$ 


\section{Data collection}

Bruker SMART 1000 CCD

diffractometer

Radiation source: fine-focus sealed tube

Graphite monochromator

$\varphi$ and $\omega$ scans

Absorption correction: multi-scan

(SADABS; Bruker, 2000)

$T_{\min }=0.846, T_{\max }=0.988$

\section{Refinement}

Refinement on $F^{2}$

Least-squares matrix: full

$R\left[F^{2}>2 \sigma\left(F^{2}\right)\right]=0.062$

$w R\left(F^{2}\right)=0.175$

$S=1.03$

5688 reflections

419 parameters

0 restraints

Primary atom site location: structure-invariant direct methods
9227 measured reflections

5688 independent reflections

3102 reflections with $I>2 \sigma(I)$

$R_{\text {int }}=0.038$

$\theta_{\max }=26.0^{\circ}, \theta_{\min }=2.1^{\circ}$

$h=-13 \rightarrow 13$

$k=-14 \rightarrow 13$

$l=-15 \rightarrow 13$

Secondary atom site location: difference Fourier map

Hydrogen site location: inferred from

neighbouring sites

$\mathrm{H}$-atom parameters constrained

$w=1 /\left[\sigma^{2}\left(F_{\mathrm{o}}^{2}\right)+(0.0681 P)^{2}+0.0868 P\right]$

where $P=\left(F_{\mathrm{o}}{ }^{2}+2 F_{\mathrm{c}}{ }^{2}\right) / 3$

$(\Delta / \sigma)_{\max }<0.001$

$\Delta \rho_{\max }=0.41 \mathrm{e}^{-3}$

$\Delta \rho_{\min }=-0.42 \mathrm{e}^{-3}$

Special details

Geometry. All e.s.d.'s (except the e.s.d. in the dihedral angle between two 1.s. planes) are estimated using the full covariance matrix. The cell e.s.d.'s are taken into account individually in the estimation of e.s.d.'s in distances, angles and torsion angles; correlations between e.s.d.'s in cell parameters are only used when they are defined by crystal symmetry. An approximate (isotropic) treatment of cell e.s.d.'s is used for estimating e.s.d.'s involving 1.s. planes.

Refinement. Refinement of $F^{2}$ against ALL reflections. The weighted $R$-factor $w R$ and goodness of fit $S$ are based on $F^{2}$, conventional $R$-factors $R$ are based on $F$, with $F$ set to zero for negative $F^{2}$. The threshold expression of $F^{2}>\sigma\left(F^{2}\right)$ is used only for calculating $R$-factors(gt) etc. and is not relevant to the choice of reflections for refinement. $R$-factors based on $F^{2}$ are statistically about twice as large as those based on $F$, and $R$ - factors based on ALL data will be even larger.

Fractional atomic coordinates and isotropic or equivalent isotropic displacement parameters $\left(\AA^{2}\right)$

\begin{tabular}{lllll}
\hline & $x$ & $y$ & $z$ & $U_{\text {iso }} * U_{\text {eq }}$ \\
\hline O1 & $1.1867(3)$ & $-0.08637(18)$ & $0.09431(18)$ & $0.0448(6)$ \\
$\mathrm{H} 1 \mathrm{O}$ & 1.1937 & -0.0154 & 0.0928 & $0.067^{*}$ \\
$\mathrm{O} 2$ & $0.8123(3)$ & $-0.2527(2)$ & $0.6061(2)$ & $0.0621(8)$ \\
$\mathrm{O} 3$ & $0.8574(3)$ & $-0.4164(2)$ & $0.5510(2)$ & $0.0703(8)$ \\
$\mathrm{O} 4$ & $0.8089(3)$ & $-0.13330(19)$ & $0.10667(19)$ & $0.0595(8)$ \\
$\mathrm{H} 4 \mathrm{O}$ & 0.7975 & -0.0622 & 0.0925 & $0.089 *$ \\
O5 & $0.4797(3)$ & $-0.2368(2)$ & $0.6310(2)$ & $0.0608(8)$ \\
O6 & $0.4974(3)$ & $-0.4093(2)$ & $0.5958(2)$ & $0.0658(8)$ \\
O7 & $0.7816(3)$ & $0.09156(19)$ & $0.4737(2)$ & $0.0502(7)$ \\
$\mathrm{H} 7 \mathrm{O}$ & 0.8186 & 0.1513 & 0.4252 & $0.075 *$ \\
O8 & $0.4783(3)$ & $0.3083(3)$ & $0.9032(2)$ & $0.0830(10)$ \\
O9 & $0.4522(3)$ & $0.1281(3)$ & $0.9805(2)$ & $0.0913(11)$ \\
$\mathrm{N} 1$ & $0.9339(3)$ & $0.2871(2)$ & $0.1376(2)$ & $0.0324(6)$ \\
$\mathrm{N} 2$ & $1.1352(3)$ & $0.1102(2)$ & $0.1657(2)$ & $0.0352(6)$ \\
$\mathrm{N} 3$ & $0.8708(3)$ & $-0.3096(3)$ & $0.5314(3)$ & $0.0458(7)$ \\
$\mathrm{N} 4$ & $0.7479(3)$ & $0.0770(2)$ & $0.1510(2)$ & $0.0354(7)$
\end{tabular}




\begin{tabular}{|c|c|c|c|c|}
\hline N5 & $0.5246(3)$ & $-0.3041(3)$ & $0.5642(3)$ & $0.0455(7)$ \\
\hline N6 & $0.8372(3)$ & $0.3109(2)$ & $0.3871(2)$ & $0.0361(7)$ \\
\hline N7 & 0.4978 (3) & $0.2066(3)$ & 0.8974 (3) & $0.0566(9)$ \\
\hline $\mathrm{C} 1$ & $1.0834(3)$ & 0.3037 (3) & 0.0811 (3) & $0.0381(8)$ \\
\hline $\mathrm{H} 1 \mathrm{~A}$ & 1.1088 & 0.3867 & 0.0680 & $0.046^{*}$ \\
\hline H1B & 1.1120 & 0.2813 & 0.0052 & $0.046^{*}$ \\
\hline $\mathrm{C} 2$ & 1.1598 (3) & $0.2351(2)$ & $0.1490(3)$ & $0.0381(8)$ \\
\hline $\mathrm{H} 2 \mathrm{~A}$ & 1.2583 & 0.2544 & 0.1084 & $0.046^{*}$ \\
\hline H2B & 1.1319 & 0.2575 & 0.2248 & $0.046^{*}$ \\
\hline C3 & $1.0620(3)$ & $0.0478(3)$ & 0.2639 (3) & $0.0336(8)$ \\
\hline H3 & 1.0176 & 0.0862 & 0.3256 & $0.040 *$ \\
\hline $\mathrm{C} 4$ & $1.0438(3)$ & $-0.0738(3)$ & 0.2849 (3) & $0.0308(7)$ \\
\hline $\mathrm{C} 5$ & $1.1155(3)$ & $-0.1371(3)$ & 0.1946 (3) & $0.0324(8)$ \\
\hline C6 & $1.1040(3)$ & $-0.2603(3)$ & $0.2275(3)$ & $0.0389(8)$ \\
\hline H6 & 1.1518 & -0.3051 & 0.1721 & $0.047 *$ \\
\hline $\mathrm{C} 7$ & $1.0271(3)$ & $-0.3153(3)$ & 0.3355 (3) & $0.0377(8)$ \\
\hline H7 & 1.0223 & -0.3971 & 0.3547 & $0.045^{*}$ \\
\hline $\mathrm{C} 8$ & 0.9544 (3) & $-0.2504(3)$ & $0.4190(3)$ & $0.0343(8)$ \\
\hline C9 & $0.9646(3)$ & $-0.1321(3)$ & 0.3945 (3) & $0.0323(7)$ \\
\hline H9 & 0.9175 & -0.0898 & 0.4524 & $0.039 *$ \\
\hline $\mathrm{C} 10$ & 0.8749 (3) & $0.2637(3)$ & $0.0560(3)$ & $0.0366(8)$ \\
\hline $\mathrm{H} 10 \mathrm{~A}$ & 0.9375 & 0.2169 & 0.0044 & $0.044^{*}$ \\
\hline H10B & 0.8665 & 0.3378 & 0.0083 & $0.044^{*}$ \\
\hline $\mathrm{C} 11$ & $0.7373(3)$ & 0.2011 (3) & 0.1132 (3) & $0.0381(8)$ \\
\hline H11A & 0.6827 & 0.2347 & 0.1803 & $0.046^{*}$ \\
\hline H11B & 0.6896 & 0.2122 & 0.0589 & $0.046^{*}$ \\
\hline C12 & $0.7036(3)$ & $0.0251(3)$ & $0.2570(3)$ & $0.0345(8)$ \\
\hline H12 & 0.6720 & 0.0712 & 0.3140 & $0.041 *$ \\
\hline $\mathrm{C} 13$ & 0.6991 (3) & $-0.0966(3)$ & $0.2943(3)$ & $0.0317(7)$ \\
\hline C14 & $0.7506(4)$ & $-0.1716(3)$ & $0.2118(3)$ & $0.0388(8)$ \\
\hline $\mathrm{C} 15$ & $0.7267(4)$ & $-0.2933(3)$ & $0.2577(3)$ & $0.0448(9)$ \\
\hline H15 & 0.7619 & -0.3456 & 0.2071 & $0.054 *$ \\
\hline C16 & $0.6556(3)$ & $-0.3364(3)$ & $0.3706(3)$ & $0.0401(8)$ \\
\hline H16 & 0.6389 & -0.4173 & 0.3977 & $0.048^{*}$ \\
\hline $\mathrm{C} 17$ & 0.6071 (3) & $-0.2591(3)$ & $0.4471(3)$ & $0.0329(8)$ \\
\hline $\mathrm{C} 18$ & $0.6295(3)$ & $-0.1425(3)$ & $0.4103(3)$ & $0.0329(8)$ \\
\hline H18 & 0.5977 & -0.0925 & 0.4637 & $0.039 *$ \\
\hline C19 & $0.8723(4)$ & $0.3882(3)$ & 0.1849 (3) & $0.0386(8)$ \\
\hline H19A & 0.7726 & 0.3806 & 0.2069 & $0.046^{*}$ \\
\hline H19B & 0.9062 & 0.4583 & 0.1245 & $0.046^{*}$ \\
\hline $\mathrm{C} 20$ & 0.9027 (4) & $0.4034(3)$ & 0.2889 (3) & $0.0394(8)$ \\
\hline $\mathrm{H} 20 \mathrm{~A}$ & 1.0021 & 0.4043 & 0.2694 & $0.047 *$ \\
\hline H20B & 0.8703 & 0.4785 & 0.3093 & $0.047^{*}$ \\
\hline $\mathrm{C} 21$ & $0.7671(3)$ & $0.3281(3)$ & 0.4904 (3) & $0.0359(8)$ \\
\hline $\mathrm{H} 21$ & 0.7578 & 0.4052 & 0.5025 & $0.043 *$ \\
\hline $\mathrm{C} 22$ & 0.7048 (3) & $0.2381(3)$ & $0.5843(3)$ & $0.0344(8)$ \\
\hline $\mathrm{C} 23$ & $0.7173(3)$ & 0.1188 (3) & $0.5718(3)$ & $0.0378(8)$ \\
\hline C24 & $0.6567(3)$ & $0.0327(3)$ & $0.6723(3)$ & $0.0436(9)$ \\
\hline
\end{tabular}




$\begin{array}{lllll}\mathrm{H} 24 & 0.6650 & -0.0463 & 0.6666 & 0.052^{*} \\ \mathrm{C} 25 & 0.5872(3) & 0.0604(3) & 0.7763(3) & 0.0446(9) \\ \mathrm{H} 25 & 0.5484 & 0.0012 & 0.8421 & 0.054^{*} \\ \mathrm{C} 26 & 0.5731(3) & 0.1773(3) & 0.7858(3) & 0.0419(9) \\ \mathrm{C} 27 & 0.6304(3) & 0.2637(3) & 0.6931(3) & 0.0400(8) \\ \mathrm{H} 27 & 0.6201 & 0.3419 & 0.7018 & 0.048^{*} \\ \mathrm{~N} 8 & 0.2620(4) & 0.5168(3) & 0.1139(4) & 0.0889(14) \\ \mathrm{C} 28 & 0.4516(4) & 0.4093(4) & 0.1626(4) & 0.0712(13) \\ \mathrm{H} 28 \mathrm{~A} & 0.4123 & 0.3395 & 0.2205 & 0.107^{*} \\ \mathrm{H} 28 \mathrm{~B} & 0.4975 & 0.4587 & 0.1924 & 0.107^{*} \\ \mathrm{H} 28 \mathrm{C} & 0.5173 & 0.3877 & 0.0931 & 0.107^{*} \\ \mathrm{C} 29 & 0.3459(4) & 0.4707(3) & 0.1358(3) & 0.0549(10) \\ \end{array}$

Atomic displacement parameters $\left(\AA^{2}\right)$

\begin{tabular}{|c|c|c|c|c|c|c|}
\hline & $U^{11}$ & $U^{22}$ & $U^{33}$ & $U^{12}$ & $U^{13}$ & $U^{23}$ \\
\hline $\mathrm{O} 1$ & $0.0633(17)$ & $0.0335(13)$ & $0.0328(14)$ & $0.0035(13)$ & $-0.0115(12)$ & $-0.0087(11)$ \\
\hline $\mathrm{O} 2$ & $0.072(2)$ & $0.0692(18)$ & $0.0320(15)$ & $-0.0026(15)$ & $-0.0059(13)$ & $-0.0066(14)$ \\
\hline $\mathrm{O} 3$ & $0.084(2)$ & $0.0460(17)$ & $0.0602(19)$ & $-0.0093(15)$ & $-0.0123(16)$ & $0.0112(15)$ \\
\hline O4 & $0.094(2)$ & $0.0395(14)$ & $0.0328(15)$ & $0.0145(15)$ & $-0.0087(14)$ & $-0.0099(12)$ \\
\hline $\mathrm{O} 5$ & $0.0694(19)$ & $0.0594(17)$ & $0.0357(15)$ & $0.0137(14)$ & $0.0020(13)$ & $-0.0127(14)$ \\
\hline O6 & $0.084(2)$ & $0.0493(17)$ & $0.0494(17)$ & $-0.0176(15)$ & $-0.0115(15)$ & $0.0005(14)$ \\
\hline $\mathrm{O} 7$ & $0.0633(18)$ & $0.0406(14)$ & $0.0415(15)$ & $0.0102(13)$ & $-0.0127(13)$ & $-0.0110(12)$ \\
\hline $\mathrm{O} 8$ & $0.103(3)$ & $0.076(2)$ & $0.0479(18)$ & $-0.0262(19)$ & $0.0065(16)$ & $-0.0275(17)$ \\
\hline O9 & $0.087(2)$ & $0.101(2)$ & $0.0378(17)$ & $0.029(2)$ & $0.0163(16)$ & $0.0149(17)$ \\
\hline N1 & $0.0443(17)$ & $0.0281(14)$ & $0.0238(14)$ & $0.0091(12)$ & $-0.0097(13)$ & $-0.0097(12)$ \\
\hline N2 & $0.0330(16)$ & $0.0322(15)$ & $0.0364(16)$ & $0.0019(13)$ & $-0.0102(13)$ & $-0.0029(13)$ \\
\hline N3 & $0.0440(19)$ & $0.054(2)$ & $0.0355(18)$ & $-0.0034(16)$ & $-0.0133(15)$ & $-0.0012(16)$ \\
\hline N4 & $0.0418(17)$ & $0.0320(15)$ & $0.0285(15)$ & $0.0032(13)$ & $-0.0096(13)$ & $-0.0040(13)$ \\
\hline N5 & $0.0449(19)$ & $0.0459(19)$ & $0.0407(18)$ & $0.0015(16)$ & $-0.0109(15)$ & $-0.0072(16)$ \\
\hline N6 & $0.0416(17)$ & $0.0404(16)$ & $0.0278(15)$ & $0.0046(13)$ & $-0.0122(13)$ & $-0.0117(13)$ \\
\hline N7 & $0.041(2)$ & $0.080(3)$ & $0.039(2)$ & $-0.0021(19)$ & $-0.0043(16)$ & $-0.010(2)$ \\
\hline $\mathrm{C} 1$ & $0.046(2)$ & $0.0312(18)$ & $0.0274(18)$ & $-0.0015(16)$ & $-0.0009(16)$ & $-0.0082(15)$ \\
\hline $\mathrm{C} 2$ & $0.042(2)$ & $0.0310(18)$ & $0.039(2)$ & $0.0011(16)$ & $-0.0113(17)$ & $-0.0085(16)$ \\
\hline $\mathrm{C} 3$ & 0.0307 (19) & $0.0388(19)$ & $0.0314(18)$ & $0.0094(15)$ & $-0.0090(15)$ & $-0.0130(16)$ \\
\hline $\mathrm{C} 4$ & $0.0327(19)$ & $0.0322(18)$ & $0.0291(18)$ & $0.0045(15)$ & $-0.0124(15)$ & $-0.0086(15)$ \\
\hline $\mathrm{C} 5$ & $0.0358(19)$ & $0.0339(18)$ & $0.0292(19)$ & $0.0031(15)$ & $-0.0150(16)$ & $-0.0044(16)$ \\
\hline C6 & $0.045(2)$ & $0.0383(19)$ & $0.038(2)$ & $0.0089(17)$ & $-0.0175(17)$ & $-0.0149(17)$ \\
\hline $\mathrm{C} 7$ & $0.050(2)$ & $0.0295(17)$ & $0.042(2)$ & $0.0021(16)$ & $-0.0262(19)$ & $-0.0080(16)$ \\
\hline $\mathrm{C} 8$ & $0.0319(19)$ & $0.042(2)$ & $0.0293(18)$ & $-0.0027(16)$ & $-0.0135(15)$ & $-0.0030(16)$ \\
\hline C9 & $0.0291(18)$ & $0.0388(19)$ & $0.0294(18)$ & $0.0076(15)$ & $-0.0106(15)$ & $-0.0087(15)$ \\
\hline $\mathrm{C} 10$ & $0.050(2)$ & $0.0351(18)$ & $0.0230(17)$ & $0.0054(16)$ & $-0.0114(16)$ & $-0.0071(15)$ \\
\hline C11 & $0.048(2)$ & $0.0372(19)$ & $0.0299(18)$ & $0.0109(17)$ & $-0.0148(17)$ & $-0.0092(16)$ \\
\hline $\mathrm{C} 12$ & $0.0306(19)$ & $0.041(2)$ & $0.036(2)$ & $0.0067(15)$ & $-0.0126(16)$ & $-0.0181(17)$ \\
\hline $\mathrm{C} 13$ & $0.0313(19)$ & $0.0330(18)$ & $0.0311(18)$ & $0.0050(15)$ & $-0.0101(15)$ & $-0.0106(15)$ \\
\hline C14 & $0.045(2)$ & $0.040(2)$ & $0.032(2)$ & $0.0098(17)$ & $-0.0143(17)$ & $-0.0088(17)$ \\
\hline $\mathrm{C} 15$ & $0.059(2)$ & $0.038(2)$ & $0.038(2)$ & $0.0122(18)$ & $-0.0147(19)$ & $-0.0171(17)$ \\
\hline $\mathrm{C} 16$ & $0.045(2)$ & $0.0348(19)$ & $0.043(2)$ & $0.0058(16)$ & $-0.0190(18)$ & $-0.0093(17)$ \\
\hline
\end{tabular}




\begin{tabular}{lllllll}
$\mathrm{C} 17$ & $0.0287(19)$ & $0.0395(19)$ & $0.0290(18)$ & $0.0020(15)$ & $-0.0091(15)$ & $-0.0068(16)$ \\
$\mathrm{C} 18$ & $0.0311(19)$ & $0.0385(19)$ & $0.0328(19)$ & $0.0054(15)$ & $-0.0123(15)$ & $-0.0152(16)$ \\
$\mathrm{C} 19$ & $0.057(2)$ & $0.0314(18)$ & $0.0251(18)$ & $0.0093(16)$ & $-0.0133(16)$ & $-0.0057(15)$ \\
$\mathrm{C} 20$ & $0.050(2)$ & $0.0349(19)$ & $0.0307(19)$ & $0.0044(17)$ & $-0.0093(16)$ & $-0.0114(16)$ \\
$\mathrm{C} 21$ & $0.040(2)$ & $0.0373(19)$ & $0.034(2)$ & $0.0062(16)$ & $-0.0170(17)$ & $-0.0110(16)$ \\
$\mathrm{C} 22$ & $0.0310(19)$ & $0.041(2)$ & $0.036(2)$ & $0.0057(15)$ & $-0.0157(16)$ & $-0.0123(17)$ \\
$\mathrm{C} 23$ & $0.0314(19)$ & $0.046(2)$ & $0.039(2)$ & $0.0077(16)$ & $-0.0170(17)$ & $-0.0101(18)$ \\
$\mathrm{C} 24$ & $0.037(2)$ & $0.042(2)$ & $0.050(2)$ & $0.0075(17)$ & $-0.0165(18)$ & $-0.0051(19)$ \\
$\mathrm{C} 25$ & $0.030(2)$ & $0.053(2)$ & $0.044(2)$ & $0.0003(17)$ & $-0.0125(17)$ & $0.0064(19)$ \\
$\mathrm{C} 26$ & $0.032(2)$ & $0.061(2)$ & $0.032(2)$ & $0.0016(18)$ & $-0.0102(16)$ & $-0.0096(18)$ \\
$\mathrm{C} 27$ & $0.037(2)$ & $0.047(2)$ & $0.037(2)$ & $-0.0007(17)$ & $-0.0115(17)$ & $-0.0134(18)$ \\
$\mathrm{N} 8$ & $0.091(3)$ & $0.051(2)$ & $0.151(4)$ & $0.020(2)$ & $-0.069(3)$ & $-0.033(2)$ \\
$\mathrm{C} 28$ & $0.069(3)$ & $0.075(3)$ & $0.066(3)$ & $0.011(3)$ & $-0.029(2)$ & $0.000(2)$ \\
$\mathrm{C} 29$ & $0.063(3)$ & $0.039(2)$ & $0.063(3)$ & $0.003(2)$ & $-0.022(2)$ & $-0.014(2)$ \\
& & & & & & \\
\hline
\end{tabular}

Geometric parameters $\left(\AA,^{\circ}\right)$

\begin{tabular}{|c|c|c|c|}
\hline $\mathrm{O} 1-\mathrm{C} 5$ & $1.262(4)$ & $\mathrm{C} 9-\mathrm{H} 9$ & 0.9500 \\
\hline $\mathrm{O} 1-\mathrm{H} 1 \mathrm{O}$ & 0.8400 & $\mathrm{C} 10-\mathrm{C} 11$ & $1.507(4)$ \\
\hline $\mathrm{O} 2-\mathrm{N} 3$ & $1.239(3)$ & $\mathrm{C} 10-\mathrm{H} 10 \mathrm{~A}$ & 0.9900 \\
\hline $\mathrm{O} 3-\mathrm{N} 3$ & $1.240(4)$ & $\mathrm{C} 10-\mathrm{H} 10 \mathrm{~B}$ & 0.9900 \\
\hline $\mathrm{O} 4-\mathrm{C} 14$ & $1.252(4)$ & $\mathrm{C} 11-\mathrm{H} 11 \mathrm{~A}$ & 0.9900 \\
\hline $\mathrm{O} 4-\mathrm{H} 4 \mathrm{O}$ & 0.8400 & C11-H11B & 0.9900 \\
\hline $\mathrm{O} 5-\mathrm{N} 5$ & $1.236(3)$ & $\mathrm{C} 12-\mathrm{C} 13$ & $1.422(4)$ \\
\hline $\mathrm{O} 6-\mathrm{N} 5$ & $1.238(3)$ & $\mathrm{C} 12-\mathrm{H} 12$ & 0.9500 \\
\hline $\mathrm{O} 7-\mathrm{C} 23$ & $1.286(4)$ & $\mathrm{C} 13-\mathrm{C} 18$ & $1.396(4)$ \\
\hline $\mathrm{O} 7-\mathrm{H} 7 \mathrm{O}$ & 0.8400 & $\mathrm{C} 13-\mathrm{C} 14$ & $1.450(4)$ \\
\hline O8-N7 & $1.227(4)$ & $\mathrm{C} 14-\mathrm{C} 15$ & $1.436(4)$ \\
\hline $\mathrm{O} 9-\mathrm{N} 7$ & $1.222(4)$ & $\mathrm{C} 15-\mathrm{C} 16$ & $1.361(4)$ \\
\hline $\mathrm{N} 1-\mathrm{C} 10$ & $1.470(4)$ & C15-H15 & 0.9500 \\
\hline $\mathrm{N} 1-\mathrm{C} 19$ & $1.471(4)$ & $\mathrm{C} 16-\mathrm{C} 17$ & $1.414(4)$ \\
\hline $\mathrm{N} 1-\mathrm{C} 1$ & $1.480(4)$ & $\mathrm{C} 16-\mathrm{H} 16$ & 0.9500 \\
\hline $\mathrm{N} 2-\mathrm{C} 3$ & $1.297(4)$ & $\mathrm{C} 17-\mathrm{C} 18$ & $1.366(4)$ \\
\hline $\mathrm{N} 2-\mathrm{C} 2$ & $1.466(4)$ & C18-H18 & 0.9500 \\
\hline N3-C8 & $1.432(4)$ & $\mathrm{C} 19-\mathrm{C} 20$ & $1.523(4)$ \\
\hline $\mathrm{N} 4-\mathrm{C} 12$ & $1.291(4)$ & C19-H19A & 0.9900 \\
\hline $\mathrm{N} 4-\mathrm{C} 11$ & $1.469(4)$ & C19-H19B & 0.9900 \\
\hline $\mathrm{N} 5-\mathrm{C} 17$ & $1.431(4)$ & $\mathrm{C} 20-\mathrm{H} 20 \mathrm{~A}$ & 0.9900 \\
\hline N6-C21 & $1.307(4)$ & $\mathrm{C} 20-\mathrm{H} 20 \mathrm{~B}$ & 0.9900 \\
\hline N6-C20 & $1.460(4)$ & $\mathrm{C} 21-\mathrm{C} 22$ & $1.406(4)$ \\
\hline N7-C26 & $1.458(4)$ & $\mathrm{C} 21-\mathrm{H} 21$ & 0.9500 \\
\hline $\mathrm{C} 1-\mathrm{C} 2$ & $1.514(4)$ & $\mathrm{C} 22-\mathrm{C} 27$ & $1.410(4)$ \\
\hline $\mathrm{C} 1-\mathrm{H} 1 \mathrm{~A}$ & 0.9900 & $\mathrm{C} 22-\mathrm{C} 23$ & $1.446(4)$ \\
\hline $\mathrm{C} 1-\mathrm{H} 1 \mathrm{~B}$ & 0.9900 & $\mathrm{C} 23-\mathrm{C} 24$ & $1.424(5)$ \\
\hline $\mathrm{C} 2-\mathrm{H} 2 \mathrm{~A}$ & 0.9900 & $\mathrm{C} 24-\mathrm{C} 25$ & $1.359(5)$ \\
\hline $\mathrm{C} 2-\mathrm{H} 2 \mathrm{~B}$ & 0.9900 & $\mathrm{C} 24-\mathrm{H} 24$ & 0.9500 \\
\hline $\mathrm{C} 3-\mathrm{C} 4$ & $1.416(4)$ & $\mathrm{C} 25-\mathrm{C} 26$ & $1.410(5)$ \\
\hline $\mathrm{C} 3-\mathrm{H} 3$ & 0.9500 & $\mathrm{C} 25-\mathrm{H} 25$ & 0.9500 \\
\hline
\end{tabular}




\begin{tabular}{|c|c|c|c|}
\hline $\mathrm{C} 4-\mathrm{C} 9$ & $1.393(4)$ & $\mathrm{C} 26-\mathrm{C} 27$ & $1.364(4)$ \\
\hline $\mathrm{C} 4-\mathrm{C} 5$ & $1.457(4)$ & $\mathrm{C} 27-\mathrm{H} 27$ & 0.9500 \\
\hline $\mathrm{C} 5-\mathrm{C} 6$ & $1.432(4)$ & $\mathrm{N} 8-\mathrm{C} 29$ & $1.126(5)$ \\
\hline $\mathrm{C} 6-\mathrm{C} 7$ & $1.360(4)$ & $\mathrm{C} 28-\mathrm{C} 29$ & $1.432(5)$ \\
\hline $\mathrm{C} 6-\mathrm{H} 6$ & 0.9500 & $\mathrm{C} 28-\mathrm{H} 28 \mathrm{~A}$ & 0.9800 \\
\hline $\mathrm{C} 7-\mathrm{C} 8$ & $1.413(4)$ & $\mathrm{C} 28-\mathrm{H} 28 \mathrm{~B}$ & 0.9800 \\
\hline $\mathrm{C} 7-\mathrm{H} 7$ & 0.9500 & $\mathrm{C} 28-\mathrm{H} 28 \mathrm{C}$ & 0.9800 \\
\hline $\mathrm{C} 8-\mathrm{C} 9$ & $1.372(4)$ & & \\
\hline $\mathrm{C} 5-\mathrm{O} 1-\mathrm{H} 1 \mathrm{O}$ & 109.5 & $\mathrm{H} 11 \mathrm{~A}-\mathrm{C} 11-\mathrm{H} 11 \mathrm{~B}$ & 107.9 \\
\hline $\mathrm{C} 14-\mathrm{O} 4-\mathrm{H} 4 \mathrm{O}$ & 109.5 & $\mathrm{~N} 4-\mathrm{C} 12-\mathrm{C} 13$ & $124.4(3)$ \\
\hline $\mathrm{C} 23-\mathrm{O} 7-\mathrm{H} 7 \mathrm{O}$ & 109.5 & $\mathrm{~N} 4-\mathrm{C} 12-\mathrm{H} 12$ & 117.8 \\
\hline $\mathrm{C} 10-\mathrm{N} 1-\mathrm{C} 19$ & $109.5(2)$ & $\mathrm{C} 13-\mathrm{C} 12-\mathrm{H} 12$ & 117.8 \\
\hline $\mathrm{C} 10-\mathrm{N} 1-\mathrm{C} 1$ & $110.2(2)$ & $\mathrm{C} 18-\mathrm{C} 13-\mathrm{C} 12$ & $118.3(3)$ \\
\hline $\mathrm{C} 19-\mathrm{N} 1-\mathrm{C} 1$ & $111.7(2)$ & $\mathrm{C} 18-\mathrm{C} 13-\mathrm{C} 14$ & $120.7(3)$ \\
\hline $\mathrm{C} 3-\mathrm{N} 2-\mathrm{C} 2$ & $122.7(3)$ & $\mathrm{C} 12-\mathrm{C} 13-\mathrm{C} 14$ & $120.6(3)$ \\
\hline $\mathrm{O} 2-\mathrm{N} 3-\mathrm{O} 3$ & $121.7(3)$ & $\mathrm{O} 4-\mathrm{C} 14-\mathrm{C} 15$ & $121.7(3)$ \\
\hline $\mathrm{O} 2-\mathrm{N} 3-\mathrm{C} 8$ & $119.1(3)$ & $\mathrm{O} 4-\mathrm{C} 14-\mathrm{C} 13$ & $122.4(3)$ \\
\hline $\mathrm{O} 3-\mathrm{N} 3-\mathrm{C} 8$ & $119.2(3)$ & $\mathrm{C} 15-\mathrm{C} 14-\mathrm{C} 13$ & $115.9(3)$ \\
\hline $\mathrm{C} 12-\mathrm{N} 4-\mathrm{C} 11$ & $123.9(3)$ & $\mathrm{C} 16-\mathrm{C} 15-\mathrm{C} 14$ & $122.6(3)$ \\
\hline $\mathrm{O} 5-\mathrm{N} 5-\mathrm{O} 6$ & $121.5(3)$ & $\mathrm{C} 16-\mathrm{C} 15-\mathrm{H} 15$ & 118.7 \\
\hline $\mathrm{O} 5-\mathrm{N} 5-\mathrm{C} 17$ & $119.3(3)$ & $\mathrm{C} 14-\mathrm{C} 15-\mathrm{H} 15$ & 118.7 \\
\hline $\mathrm{O} 6-\mathrm{N} 5-\mathrm{C} 17$ & $119.2(3)$ & $\mathrm{C} 15-\mathrm{C} 16-\mathrm{C} 17$ & $119.0(3)$ \\
\hline $\mathrm{C} 21-\mathrm{N} 6-\mathrm{C} 20$ & $123.8(3)$ & $\mathrm{C} 15-\mathrm{C} 16-\mathrm{H} 16$ & 120.5 \\
\hline $\mathrm{O} 9-\mathrm{N} 7-\mathrm{O} 8$ & $122.7(3)$ & $\mathrm{C} 17-\mathrm{C} 16-\mathrm{H} 16$ & 120.5 \\
\hline $\mathrm{O} 9-\mathrm{N} 7-\mathrm{C} 26$ & $118.5(4)$ & $\mathrm{C} 18-\mathrm{C} 17-\mathrm{C} 16$ & $121.7(3)$ \\
\hline $\mathrm{O} 8-\mathrm{N} 7-\mathrm{C} 26$ & $118.8(3)$ & $\mathrm{C} 18-\mathrm{C} 17-\mathrm{N} 5$ & $119.3(3)$ \\
\hline $\mathrm{N} 1-\mathrm{C} 1-\mathrm{C} 2$ & $113.8(3)$ & $\mathrm{C} 16-\mathrm{C} 17-\mathrm{N} 5$ & $118.9(3)$ \\
\hline $\mathrm{N} 1-\mathrm{C} 1-\mathrm{H} 1 \mathrm{~A}$ & 108.8 & $\mathrm{C} 17-\mathrm{C} 18-\mathrm{C} 13$ & $120.0(3)$ \\
\hline $\mathrm{C} 2-\mathrm{C} 1-\mathrm{H} 1 \mathrm{~A}$ & 108.8 & $\mathrm{C} 17-\mathrm{C} 18-\mathrm{H} 18$ & 120.0 \\
\hline $\mathrm{N} 1-\mathrm{C} 1-\mathrm{H} 1 \mathrm{~B}$ & 108.8 & $\mathrm{C} 13-\mathrm{C} 18-\mathrm{H} 18$ & 120.0 \\
\hline $\mathrm{C} 2-\mathrm{C} 1-\mathrm{H} 1 \mathrm{~B}$ & 108.8 & $\mathrm{~N} 1-\mathrm{C} 19-\mathrm{C} 20$ & $113.4(3)$ \\
\hline $\mathrm{H} 1 \mathrm{~A}-\mathrm{C} 1-\mathrm{H} 1 \mathrm{~B}$ & 107.7 & $\mathrm{~N} 1-\mathrm{C} 19-\mathrm{H} 19 \mathrm{~A}$ & 108.9 \\
\hline $\mathrm{N} 2-\mathrm{C} 2-\mathrm{C} 1$ & $112.5(3)$ & $\mathrm{C} 20-\mathrm{C} 19-\mathrm{H} 19 \mathrm{~A}$ & 108.9 \\
\hline $\mathrm{N} 2-\mathrm{C} 2-\mathrm{H} 2 \mathrm{~A}$ & 109.1 & $\mathrm{~N} 1-\mathrm{C} 19-\mathrm{H} 19 \mathrm{~B}$ & 108.9 \\
\hline $\mathrm{C} 1-\mathrm{C} 2-\mathrm{H} 2 \mathrm{~A}$ & 109.1 & $\mathrm{C} 20-\mathrm{C} 19-\mathrm{H} 19 \mathrm{~B}$ & 108.9 \\
\hline $\mathrm{N} 2-\mathrm{C} 2-\mathrm{H} 2 \mathrm{~B}$ & 109.1 & $\mathrm{H} 19 \mathrm{~A}-\mathrm{C} 19-\mathrm{H} 19 \mathrm{~B}$ & 107.7 \\
\hline $\mathrm{C} 1-\mathrm{C} 2-\mathrm{H} 2 \mathrm{~B}$ & 109.1 & $\mathrm{~N} 6-\mathrm{C} 20-\mathrm{C} 19$ & $111.5(3)$ \\
\hline $\mathrm{H} 2 \mathrm{~A}-\mathrm{C} 2-\mathrm{H} 2 \mathrm{~B}$ & 107.8 & $\mathrm{~N} 6-\mathrm{C} 20-\mathrm{H} 20 \mathrm{~A}$ & 109.3 \\
\hline $\mathrm{N} 2-\mathrm{C} 3-\mathrm{C} 4$ & $124.2(3)$ & $\mathrm{C} 19-\mathrm{C} 20-\mathrm{H} 20 \mathrm{~A}$ & 109.3 \\
\hline $\mathrm{N} 2-\mathrm{C} 3-\mathrm{H} 3$ & 117.9 & $\mathrm{~N} 6-\mathrm{C} 20-\mathrm{H} 20 \mathrm{~B}$ & 109.3 \\
\hline $\mathrm{C} 4-\mathrm{C} 3-\mathrm{H} 3$ & 117.9 & $\mathrm{C} 19-\mathrm{C} 20-\mathrm{H} 20 \mathrm{~B}$ & 109.3 \\
\hline $\mathrm{C} 9-\mathrm{C} 4-\mathrm{C} 3$ & $119.2(3)$ & $\mathrm{H} 20 \mathrm{~A}-\mathrm{C} 20-\mathrm{H} 20 \mathrm{~B}$ & 108.0 \\
\hline $\mathrm{C} 9-\mathrm{C} 4-\mathrm{C} 5$ & $120.8(3)$ & $\mathrm{N} 6-\mathrm{C} 21-\mathrm{C} 22$ & $123.3(3)$ \\
\hline $\mathrm{C} 3-\mathrm{C} 4-\mathrm{C} 5$ & $119.9(3)$ & $\mathrm{N} 6-\mathrm{C} 21-\mathrm{H} 21$ & 118.4 \\
\hline $\mathrm{O} 1-\mathrm{C} 5-\mathrm{C} 6$ & $122.0(3)$ & $\mathrm{C} 22-\mathrm{C} 21-\mathrm{H} 21$ & 118.4 \\
\hline $\mathrm{O} 1-\mathrm{C} 5-\mathrm{C} 4$ & $122.0(3)$ & $\mathrm{C} 21-\mathrm{C} 22-\mathrm{C} 27$ & $119.9(3)$ \\
\hline $\mathrm{C} 6-\mathrm{C} 5-\mathrm{C} 4$ & $116.0(3)$ & $\mathrm{C} 21-\mathrm{C} 22-\mathrm{C} 23$ & $121.0(3)$ \\
\hline
\end{tabular}




\begin{tabular}{|c|c|}
\hline $\mathrm{C} 7-\mathrm{C} 6-\mathrm{C} 5$ & $122.2(3)$ \\
\hline $\mathrm{C} 7-\mathrm{C} 6-\mathrm{H} 6$ & 118.9 \\
\hline $\mathrm{C} 5-\mathrm{C} 6-\mathrm{H} 6$ & 118.9 \\
\hline $\mathrm{C} 6-\mathrm{C} 7-\mathrm{C} 8$ & $119.9(3)$ \\
\hline $\mathrm{C} 6-\mathrm{C} 7-\mathrm{H} 7$ & 120.0 \\
\hline $\mathrm{C} 8-\mathrm{C} 7-\mathrm{H} 7$ & 120.0 \\
\hline $\mathrm{C} 9-\mathrm{C} 8-\mathrm{C} 7$ & $121.0(3)$ \\
\hline $\mathrm{C} 9-\mathrm{C} 8-\mathrm{N} 3$ & $119.8(3)$ \\
\hline $\mathrm{C} 7-\mathrm{C} 8-\mathrm{N} 3$ & $119.3(3)$ \\
\hline $\mathrm{C} 8-\mathrm{C} 9-\mathrm{C} 4$ & $120.1(3)$ \\
\hline $\mathrm{C} 8-\mathrm{C} 9-\mathrm{H} 9$ & 119.9 \\
\hline $\mathrm{C} 4-\mathrm{C} 9-\mathrm{H} 9$ & 119.9 \\
\hline $\mathrm{N} 1-\mathrm{C} 10-\mathrm{C} 11$ & $113.3(3)$ \\
\hline $\mathrm{N} 1-\mathrm{C} 10-\mathrm{H} 10 \mathrm{~A}$ & 108.9 \\
\hline $\mathrm{C} 11-\mathrm{C} 10-\mathrm{H} 10 \mathrm{~A}$ & 108.9 \\
\hline $\mathrm{N} 1-\mathrm{C} 10-\mathrm{H} 10 \mathrm{~B}$ & 108.9 \\
\hline $\mathrm{C} 11-\mathrm{C} 10-\mathrm{H} 10 \mathrm{~B}$ & 108.9 \\
\hline $\mathrm{H} 10 \mathrm{~A}-\mathrm{C} 10-\mathrm{H} 10 \mathrm{~B}$ & 107.7 \\
\hline $\mathrm{N} 4-\mathrm{C} 11-\mathrm{C} 10$ & $111.9(3)$ \\
\hline $\mathrm{N} 4-\mathrm{C} 11-\mathrm{H} 11 \mathrm{~A}$ & 109.2 \\
\hline $\mathrm{C} 10-\mathrm{C} 11-\mathrm{H} 11 \mathrm{~A}$ & 109.2 \\
\hline $\mathrm{N} 4-\mathrm{C} 11-\mathrm{H} 11 \mathrm{~B}$ & 109.2 \\
\hline $\mathrm{C} 10-\mathrm{C} 11-\mathrm{H} 11 \mathrm{~B}$ & 109.2 \\
\hline $\mathrm{C} 10-\mathrm{N} 1-\mathrm{C} 1-\mathrm{C} 2$ & $-131.8(3)$ \\
\hline $\mathrm{C} 19-\mathrm{N} 1-\mathrm{C} 1-\mathrm{C} 2$ & $106.1(3)$ \\
\hline $\mathrm{C} 3-\mathrm{N} 2-\mathrm{C} 2-\mathrm{C} 1$ & $-107.8(3)$ \\
\hline $\mathrm{N} 1-\mathrm{C} 1-\mathrm{C} 2-\mathrm{N} 2$ & $62.4(3)$ \\
\hline $\mathrm{C} 2-\mathrm{N} 2-\mathrm{C} 3-\mathrm{C} 4$ & $-175.4(3)$ \\
\hline $\mathrm{N} 2-\mathrm{C} 3-\mathrm{C} 4-\mathrm{C} 9$ & $178.9(3)$ \\
\hline $\mathrm{N} 2-\mathrm{C} 3-\mathrm{C} 4-\mathrm{C} 5$ & $3.2(5)$ \\
\hline $\mathrm{C} 9-\mathrm{C} 4-\mathrm{C} 5-\mathrm{O} 1$ & $179.2(3)$ \\
\hline $\mathrm{C} 3-\mathrm{C} 4-\mathrm{C} 5-\mathrm{O} 1$ & $-5.1(5)$ \\
\hline $\mathrm{C} 9-\mathrm{C} 4-\mathrm{C} 5-\mathrm{C} 6$ & $-2.9(4)$ \\
\hline $\mathrm{C} 3-\mathrm{C} 4-\mathrm{C} 5-\mathrm{C} 6$ & $172.7(3)$ \\
\hline $\mathrm{O} 1-\mathrm{C} 5-\mathrm{C} 6-\mathrm{C} 7$ & $-179.9(3)$ \\
\hline $\mathrm{C} 4-\mathrm{C} 5-\mathrm{C} 6-\mathrm{C} 7$ & $2.3(4)$ \\
\hline $\mathrm{C} 5-\mathrm{C} 6-\mathrm{C} 7-\mathrm{C} 8$ & $0.5(5)$ \\
\hline $\mathrm{C} 6-\mathrm{C} 7-\mathrm{C} 8-\mathrm{C} 9$ & $-2.8(5)$ \\
\hline $\mathrm{C} 6-\mathrm{C} 7-\mathrm{C} 8-\mathrm{N} 3$ & $178.3(3)$ \\
\hline $\mathrm{O} 2-\mathrm{N} 3-\mathrm{C} 8-\mathrm{C} 9$ & $-2.3(4)$ \\
\hline $\mathrm{O} 3-\mathrm{N} 3-\mathrm{C} 8-\mathrm{C} 9$ & $176.7(3)$ \\
\hline $\mathrm{O} 2-\mathrm{N} 3-\mathrm{C} 8-\mathrm{C} 7$ & $176.6(3)$ \\
\hline $\mathrm{O} 3-\mathrm{N} 3-\mathrm{C} 8-\mathrm{C} 7$ & $-4.5(4)$ \\
\hline $\mathrm{C} 7-\mathrm{C} 8-\mathrm{C} 9-\mathrm{C} 4$ & $2.2(4)$ \\
\hline $\mathrm{N} 3-\mathrm{C} 8-\mathrm{C} 9-\mathrm{C} 4$ & $-179.0(3)$ \\
\hline $\mathrm{C} 3-\mathrm{C} 4-\mathrm{C} 9-\mathrm{C} 8$ & $-174.9(3)$ \\
\hline $\mathrm{C} 5-\mathrm{C} 4-\mathrm{C} 9-\mathrm{C} 8$ & $0.8(4)$ \\
\hline
\end{tabular}

$\begin{array}{ll}\mathrm{C} 27-\mathrm{C} 22-\mathrm{C} 23 & 119.1(3) \\ \mathrm{O} 7-\mathrm{C} 23-\mathrm{C} 24 & 121.3(3) \\ \mathrm{O} 7-\mathrm{C} 23-\mathrm{C} 22 & 121.2(3) \\ \mathrm{C} 24-\mathrm{C} 23-\mathrm{C} 22 & 117.5(3) \\ \mathrm{C} 25-\mathrm{C} 24-\mathrm{C} 23 & 121.9(3) \\ \mathrm{C} 25-\mathrm{C} 24-\mathrm{H} 24 & 119.1 \\ \mathrm{C} 23-\mathrm{C} 24-\mathrm{H} 24 & 119.1 \\ \mathrm{C} 24-\mathrm{C} 25-\mathrm{C} 26 & 119.6(3) \\ \mathrm{C} 24-\mathrm{C} 25-\mathrm{H} 25 & 120.2 \\ \mathrm{C} 26-\mathrm{C} 25-\mathrm{H} 25 & 120.2 \\ \mathrm{C} 27-\mathrm{C} 26-\mathrm{C} 25 & 121.3(3) \\ \mathrm{C} 27-\mathrm{C} 26-\mathrm{N} 7 & 119.3(3) \\ \mathrm{C} 25-\mathrm{C} 26-\mathrm{N} 7 & 119.5(3) \\ \mathrm{C} 26-\mathrm{C} 27-\mathrm{C} 22 & 120.6(3) \\ \mathrm{C} 26-\mathrm{C} 27-\mathrm{H} 27 & 119.7\end{array}$

$\mathrm{C} 26-\mathrm{C} 27-\mathrm{H} 27 \quad 119.7$

$\mathrm{C} 22-\mathrm{C} 27-\mathrm{H} 27 \quad 119.7$

$\mathrm{C} 29-\mathrm{C} 28-\mathrm{H} 28 \mathrm{~A} \quad 109.5$

$\mathrm{C} 29-\mathrm{C} 28-\mathrm{H} 28 \mathrm{~B} \quad 109.5$

$\mathrm{H} 28 \mathrm{~A}-\mathrm{C} 28-\mathrm{H} 28 \mathrm{~B} \quad 109.5$

$\mathrm{C} 29-\mathrm{C} 28-\mathrm{H} 28 \mathrm{C} \quad 109.5$

$\mathrm{H} 28 \mathrm{~A}-\mathrm{C} 28-\mathrm{H} 28 \mathrm{C} \quad 109.5$

$\mathrm{H} 28 \mathrm{~B}-\mathrm{C} 28-\mathrm{H} 28 \mathrm{C} \quad 109.5$

$\mathrm{N} 8-\mathrm{C} 29-\mathrm{C} 28$

$\mathrm{C} 13-\mathrm{C} 14-\mathrm{C} 15-\mathrm{C} 16 \quad 2.5(5)$

$\mathrm{C} 14-\mathrm{C} 15-\mathrm{C} 16-\mathrm{C} 17 \quad-2.2(5)$

$\mathrm{C} 15-\mathrm{C} 16-\mathrm{C} 17-\mathrm{C} 18 \quad 0.1(5)$

$\mathrm{C} 15-\mathrm{C} 16-\mathrm{C} 17-\mathrm{N} 5 \quad 175.8(3)$

$\mathrm{O} 5-\mathrm{N} 5-\mathrm{C} 17-\mathrm{C} 18 \quad-2.8$ (4)

$\mathrm{O} 6-\mathrm{N} 5-\mathrm{C} 17-\mathrm{C} 18 \quad 175.5$ (3)

$\mathrm{O} 5-\mathrm{N} 5-\mathrm{C} 17-\mathrm{C} 16 \quad-178.6(3)$

$\mathrm{O} 6-\mathrm{N} 5-\mathrm{C} 17-\mathrm{C} 16 \quad-0.3(4)$

$\mathrm{C} 16-\mathrm{C} 17-\mathrm{C} 18-\mathrm{C} 13$

N5-C17-C18-C13 -174.1 (3)

$\mathrm{C} 12-\mathrm{C} 13-\mathrm{C} 18-\mathrm{C} 17 \quad 171.2(3)$

$\mathrm{C} 14-\mathrm{C} 13-\mathrm{C} 18-\mathrm{C} 17 \quad-1.2(4)$

$\mathrm{C} 10-\mathrm{N} 1-\mathrm{C} 19-\mathrm{C} 20 \quad 167.1$ (3)

$\mathrm{C} 1-\mathrm{N} 1-\mathrm{C} 19-\mathrm{C} 20 \quad-70.5$ (3)

$\mathrm{C} 21-\mathrm{N} 6-\mathrm{C} 20-\mathrm{C} 19 \quad-132.3$ (3)

$\mathrm{N} 1-\mathrm{C} 19-\mathrm{C} 20-\mathrm{N} 6 \quad-67.3(4)$

$\mathrm{C} 20-\mathrm{N} 6-\mathrm{C} 21-\mathrm{C} 22 \quad-179.8(3)$

$\mathrm{N} 6-\mathrm{C} 21-\mathrm{C} 22-\mathrm{C} 27 \quad-179.8$ (3)

$\mathrm{N} 6-\mathrm{C} 21-\mathrm{C} 22-\mathrm{C} 23 \quad 1.2(5)$

$\mathrm{C} 21-\mathrm{C} 22-\mathrm{C} 23-\mathrm{O} 7 \quad-2.5(5)$

$\mathrm{C} 27-\mathrm{C} 22-\mathrm{C} 23-\mathrm{O} 7 \quad 178.4$ (3)

$\mathrm{C} 21-\mathrm{C} 22-\mathrm{C} 23-\mathrm{C} 24 \quad 177.0$ (3)

$\mathrm{C} 27-\mathrm{C} 22-\mathrm{C} 23-\mathrm{C} 24 \quad-2.0$ (4)

$\mathrm{O} 7-\mathrm{C} 23-\mathrm{C} 24-\mathrm{C} 25 \quad-179.3$ (3) 


$\begin{array}{llll}\mathrm{C} 19-\mathrm{N} 1-\mathrm{C} 10-\mathrm{C} 11 & -79.5(3) & \mathrm{C} 22-\mathrm{C} 23-\mathrm{C} 24-\mathrm{C} 25 & 1.2(5) \\ \mathrm{C} 1-\mathrm{N} 1-\mathrm{C} 10-\mathrm{C} 11 & 157.2(2) & \mathrm{C} 23-\mathrm{C} 24-\mathrm{C} 25-\mathrm{C} 26 & 0.5(5) \\ \mathrm{C} 12-\mathrm{N} 4-\mathrm{C} 11-\mathrm{C} 10 & 114.6(3) & \mathrm{C} 24-\mathrm{C} 25-\mathrm{C} 26-\mathrm{C} 27 & -1.5(5) \\ \mathrm{N} 1-\mathrm{C} 10-\mathrm{C} 11-\mathrm{N} 4 & -78.8(3) & \mathrm{C} 24-\mathrm{C} 25-\mathrm{C} 26-\mathrm{N} 7 & 179.6(3) \\ \mathrm{C} 11-\mathrm{N} 4-\mathrm{C} 12-\mathrm{C} 13 & 172.5(3) & \mathrm{O} 9-\mathrm{N} 7-\mathrm{C} 26-\mathrm{C} 27 & -176.8(3) \\ \mathrm{N} 4-\mathrm{C} 12-\mathrm{C} 13-\mathrm{C} 18 & -170.1(3) & \mathrm{O} 8-\mathrm{N} 7-\mathrm{C} 26-\mathrm{C} 27 & 6.0(5) \\ \mathrm{N} 4-\mathrm{C} 12-\mathrm{C} 13-\mathrm{C} 14 & 2.4(5) & \mathrm{O} 9-\mathrm{N} 7-\mathrm{C} 26-\mathrm{C} 25 & 2.1(5) \\ \mathrm{C} 18-\mathrm{C} 13-\mathrm{C} 14-\mathrm{O} 4 & 177.3(3) & \mathrm{O} 8-\mathrm{N} 7-\mathrm{C} 26-\mathrm{C} 25 & -175.1(3) \\ \mathrm{C} 12-\mathrm{C} 13-\mathrm{C} 14-\mathrm{O} 4 & 5.0(5) & \mathrm{C} 25-\mathrm{C} 26-\mathrm{C} 27-\mathrm{C} 22 & 0.6(5) \\ \mathrm{C} 18-\mathrm{C} 13-\mathrm{C} 14-\mathrm{C} 15 & -0.8(4) & \mathrm{N} 7-\mathrm{C} 26-\mathrm{C} 27-\mathrm{C} 22 & 179.5(3) \\ \mathrm{C} 12-\mathrm{C} 13-\mathrm{C} 14-\mathrm{C} 15 & -173.0(3) & \mathrm{C} 21-\mathrm{C} 22-\mathrm{C} 27-\mathrm{C} 26 & -177.9(3) \\ \mathrm{O} 4-\mathrm{C} 14-\mathrm{C} 15-\mathrm{C} 16 & -175.5(3) & \mathrm{C} 23-\mathrm{C} 22-\mathrm{C} 27-\mathrm{C} 26 & 1.2(5)\end{array}$

Hydrogen-bond geometry $\left(\AA,{ }^{\circ}\right)$

\begin{tabular}{|c|c|c|c|c|}
\hline$D-\mathrm{H}^{\cdots} \cdots A$ & $D-\mathrm{H}$ & $\mathrm{H} \cdots A$ & $D \cdots A$ & $D-\mathrm{H} \cdots A$ \\
\hline $\mathrm{O} 1-\mathrm{H} 1 O^{\cdots} \cdot \mathrm{N} 2$ & 0.84 & 1.87 & $2.627(3)$ & 149 \\
\hline $\mathrm{O} 4-\mathrm{H} 4 O \cdots \mathrm{N} 4$ & 0.84 & 1.92 & $2.665(3)$ & 147 \\
\hline $\mathrm{O} 7-\mathrm{H} 7 O^{\cdots} \mathrm{N} 6$ & 0.84 & 1.85 & $2.607(3)$ & 149 \\
\hline $\mathrm{C} 1-\mathrm{H} 1 A \cdots \mathrm{N} 8^{\mathrm{i}}$ & 0.99 & 2.56 & $3.369(5)$ & 139 \\
\hline $\mathrm{C} 1-\mathrm{H} 1 B \cdots \mathrm{O} 4^{\mathrm{ii}}$ & 0.99 & 2.41 & $3.290(4)$ & 148 \\
\hline $\mathrm{C} 2-\mathrm{H} 2 B \cdots \mathrm{O} 2^{i \mathrm{iii}}$ & 0.99 & 2.44 & $3.300(4)$ & 146 \\
\hline $\mathrm{C} 3-\mathrm{H} 3 \cdots \mathrm{O} 7$ & 0.95 & 2.53 & $3.297(4)$ & 138 \\
\hline $\mathrm{C} 6-\mathrm{H} 6 \cdots \mathrm{N}^{\mathrm{iv}}$ & 0.95 & 2.49 & $3.360(5)$ & 153 \\
\hline $\mathrm{C} 9-\mathrm{H} 9 \cdots \mathrm{O} 7$ & 0.95 & 2.55 & $3.328(4)$ & 139 \\
\hline $\mathrm{C} 11-\mathrm{H} 11 A \cdots \mathrm{O}^{\mathrm{v}}$ & 0.99 & 2.40 & $3.331(4)$ & 157 \\
\hline $\mathrm{C} 12-\mathrm{H} 12 \cdots{ }^{\mathrm{O}} 5^{\mathrm{v}}$ & 0.95 & 2.54 & $3.339(4)$ & 142 \\
\hline $\mathrm{C} 16-\mathrm{H} 16 \cdots{ }^{\mathrm{O}} 6^{\mathrm{vi}}$ & 0.95 & 2.51 & $3.330(4)$ & 145 \\
\hline $\mathrm{C} 25-\mathrm{H} 25 \cdots \mathrm{O} 9^{\text {vii }}$ & 0.95 & 2.48 & $3.359(4)$ & 153 \\
\hline
\end{tabular}

Symmetry codes: (i) $x+1, y, z$; (ii) $-x+2,-y,-z$; (iii) $-x+2,-y,-z+1$; (iv) $x+1, y-1, z$; (v) $-x+1,-y,-z+1$; (vi) $-x+1,-y-1,-z+1$; (vii) $-x+1,-y,-z+2$. 